\title{
Design and Develop an Energy Efficient Methodology for Wireless Ad-hoc network
}

\author{
Minakshi Kalita \\ Department of Computer Science \& Engineering and Information Technology \\ School of Technology, Assam Don Bosco University, Guwahati-781017, INDIA \\ Bobby Sharma \\ Department of Computer Science \& Engineering and Information Technology \\ School of Technology, Assam Don Bosco University, Guwahati-781017, INDIA
}

\begin{abstract}
A Mobile Ad-Hoc Network (MANET) is an infrastructure less network where there is no centralized administrator. MANET is a collection of mobile nodes that relies on battery power. The topology is dynamic as the nodes are moving independently. Due to the mobility of each of the node in MANET, the energy consumption of each node is more, which degrades the overall performance of the network. Therefore energy consumption is the major issue in MANET. This paper proposes a new methodology for conserving energy in each of the node present in the MANET. The proposed new methodology announces energy and based on nodes sending and receiving rates and the sizes of the data to be transmitted it justifies whether its energy level is maintained or decreased. It calculates the energy level in each of the node before they are selected for routing path. In this new methodology, some threshold values are defined for Residual Energy, Number of Control traffic and Packet Size. If the node's energy level is greater than the threshold value and number of control traffic as well as packet size is smaller than the corresponding threshold values, then only the node is considered for routing; if not, then that node is directly discarded from route selection process. The proposed methodology conserves the energy in each of the node in the network by forwarding the data packets of smaller size only. The implementation and analysis is done on NS2. This methodology is mainly based on the AODV Routing Protocol.
\end{abstract}

Keywords - Ad-hoc, AODV, MANET, NS2.

\section{INTRODUCTION}

A Mobile Ad-Hoc Network (MANET) is a collection of mobile nodes that are communicating with each other without any centralized administration. Since MANET does not have any fixed infrastructure, the mobile nodes acts as a router as well as a host, i.e., each node participates in routing by forwarding data to the other nodes via the wireless links. The mobile nodes in MANET are moving independently as their topology is Dynamic in nature. One of the most important characteristics of MANET is their limited battery capacity of the nodes, i.e, energy constrained node. MANET has some unique features, such as absence of centralized control, time-varying wireless link characteristics, route changes occur due to mobility, the limited range of wireless transmission and packet losses due to the hidden terminal problem [1]. In MANET, the process of routing is complex. So, in case of determining the network performance of MANET, routing protocol plays a very crucial role.

Depending on the route discovery process, routing protocols are divided into three categories: Proactive Routing Protocol such as Destination Sequenced Distance Vector Routing (DSDV), Optimized Link State Routing (OLSR), Topology Broadcast based on Reverse Path Forwarding (TBRPF); Reactive or On-demand routing protocols such as Dynamic Source Routing (DSR), Ad-hoc on Demand Distance Vector (AODV), Signal Stability based Adaptive routing (SSA) and Hybrid protocols such as Zone Routing Protocol (ZRP), Hybrid Ad-hoc Routing protocol (HARP) [1].

On-demand routing protocols are easy to realize and their overhead is low. But routes in on-demand routing protocols are easy to break in the case of topology variations. In AODV, node doesn't have any information about other nodes until a communication is needed. After a particular interval of time, by broadcasting HELLO packets, each node collects its neighbors' connectivity information. Local connectivity maintains information about all the neighbors [4]. 


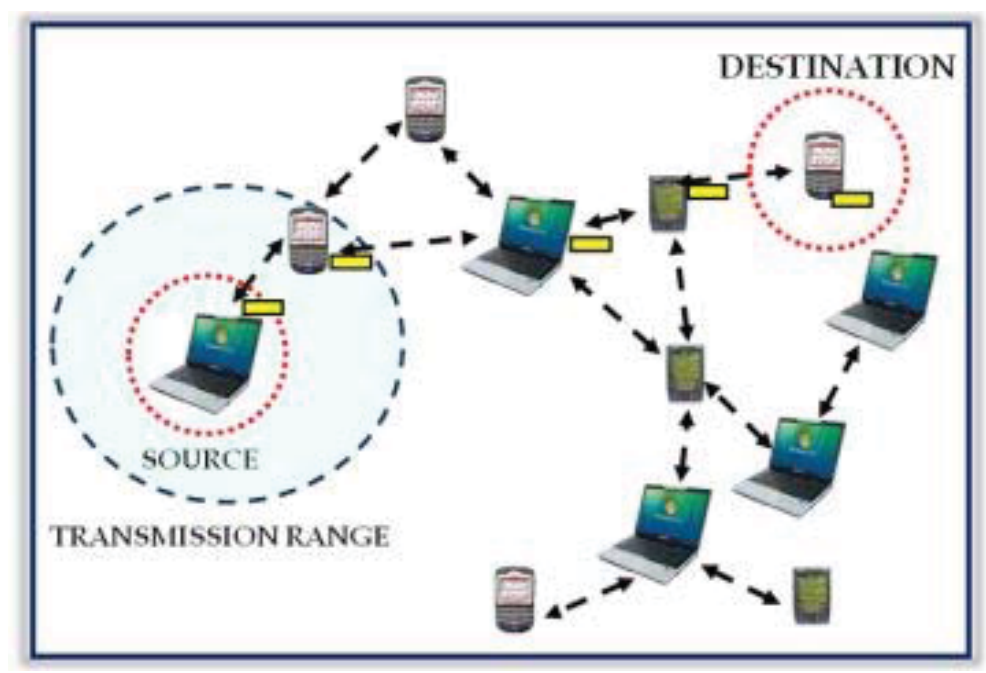

Fig. 1: Mobile Ad-hoc Network (MANET) [2]

In MANET, the nodes rely only on the battery power. Since the nodes are battery operated, the energy in each of the node need to be conserved which will prolong the lifetime of the nodes in the network, consequently the overall performance of the network is enhanced. To make the most of the lifetime of an ad hoc network, it is essential to lengthen each individual node's life through minimizing the total transmission energy consumption for each communication request, or total receiving power for each communication request and by varying the packet size. , an efficient methodology based on the AODV protocol must satisfy that the energy consumption rate at each node is evenly distributed and at the same time the total transmission energy, total receiving energy for each request is minimized [4].

\section{LITERATURE SURVEY}

As in [5], Adaptive HELLO messaging scheme determines the local link connectivity information for monitoring the link status between nodes along with the incorporation of Dynamic On Demand Routing Protocol which reduces the energy consumption of mobile nodes to certain extent. This messaging scheme suppresses the unnecessary HELLO messages without reduced delectability of broken links. If a node is not involved in any communication with any other node for a given period of time, then it need not maintain the local connectivity information by broadcasting the HELLO messages during that period. In this messaging scheme, unnecessary HELLO messages can be reduced which will conserve the energy to some extent. As a result of this, mean delay will be decreased along with good packet delivery ratio.

Efficient Power Aware Routing Protocol (EPAR) [6] is a new power aware routing protocol that increases the network lifetime by conserving the energy using min-max formulation. EPAR identifies the capacity of a node by the expected energy spent in reliably forwarding data packets over a specific link. The energy consumed for one packet is calculated by-

$$
E c=\sum_{i=1}^{k} T(n i, n i+1)
$$

where $n_{i}$ to $n_{k}$ are the nodes in the route and T denotes the energy consumed in transmitting and receiving a packet over one hop. This protocol selects the path that has the largest packet capacity at the smallest residual packet transmission capacity. The algorithm reduces for more than $20 \%$ the total energy consumption and decreases the mean delay, especially for high load networks. It produces good results in terms of throughput and leads to good packet delivery ratio.

As in [7], it describes a series of experiments which obtained detailed measurements of the energy consumption of an IEEE 802.11 wireless network interface operating in an ad hoc networking environment. These experiments show that the energy consumption of an IEEE 802.11 wireless interface has a complex range of behaviours that are relevant to the design of network layer protocols - energy consumption is not synonymous with bandwidth utilization. 
As in [8], Power-Aware Routing Protocol evenly distributed the power consumption rate of each node as a result of which the overall transmission power for each connection request is minimized. At Network Layer, the routing algorithms must select the best path to minimize the total power needed to route packets and maximize the lifetime of all the nodes. Four variations of route selection:

a. Minimum Total Transmission Power Routing (MTPR): For successful transmissions from a host ni to nj, the SNR at host $\mathrm{nj}$ is-

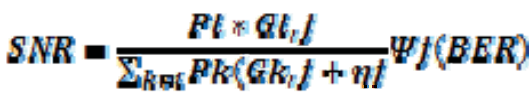

where $\mathrm{Pi}$ is the transmission power of host $\mathrm{ni}, \mathrm{Gi}, \mathrm{j}$ is the path gain between host $\mathrm{ni}$ and $\mathrm{nj}$, $\mathrm{\eta j}$ is the thermal noise at nj. Minimum Transmission Power is dependent on the interference noise, distance between hosts and bit error rate (BER). To obtain the route with minimum total power, the transmission power $\mathrm{P}(\mathrm{ni}, \mathrm{nj})$ between host ni and nj is used as a metric.

b. Minimum Battery Cost Routing (MBCR): To find the route with maximum battery capacity, we should select a route $i$ that has minimum battery cost-

$$
\mathbf{R i}=\min \{\mathbf{R j} \mid \mathbf{j} € \mathbf{A}\} \text {. }
$$

where $\mathrm{A}$ is the set containing all possible routes, $\mathrm{Rj}$ is battery cost for route $\mathrm{i}$ consisting of $\mathrm{D}$ nodes. Since battery capacity is directly incorporated into the routing protocol, this metric prevents hosts from being overused, thereby increasing the lifetime.

c. Min-Max Battery Cost Routing (MMBCR): To make sure that no node is overused; the value of $\mathrm{Rj}$ is modified as below-

\section{$\max _{\text {tarowte } f} f t\left(c^{2} t\right)$}

where $f i f\left(e^{b} t\right)$ is the battery cost function of host ni. And the desired route i can be obtained from the equation below-

$$
\mathbf{R i}=\min \{\mathbf{R j} \mid \mathbf{j} € \mathbf{A}\} .
$$

Since this nictric always tries to avoid the route with nodes having the least battery capacity among a11 nodes in all possible routes, the battery of each host will be used more fairly than in previous schemes.

d. Conditional Min-Max Battery Cost Routing (CMMBCR): By applying MTPR, MBCR and MMBCR, maximization of lifetime of each node cannot be achieved in all the conditions. So to resolve these problems, CMMBCR was proposed. In this scheme, instead of cost function, battery capacity is used as a metric

As in [9], Minimum Energy Dynamic Source Routing (MEDSR) Protocol for MANET ensures the connectivity of the network, which consists of two mechanism- i) route discovery ii). Link power adjustment. This protocol saves a considerable portion of per packet energy consumption.

As in [11], Energy Efficient Dynamic Source Routing (EEDSR) protocol satisfies less energy consumption from the viewpoints of nodes and networks. This protocol reduces the total energy consumption and decreases the mean delay, especially for high load networks, while achieving a good packet delivery ratio. The simulation result shows that it reduces $10 \%$ of total energy consumption and decreases mean delay.

As in [12], LEACH generate cluster and gives information about energy of each cluster belongs zone and if energy of the node is higher so LEACH select that particular node for data transmission that work increases the reliability to the communication. The analysis result is in the form of network parameter like throughput, packet delivery ratio, energy consumption via node and routing overhead.

As in [13], the Energy Efficient Ad hoc On Demand location based Routing Protocol (EAODVDREAM) improves the routing performance and energy utilization in Wireless Ad hoc Network. This protocol combines the positionbased routing features into On demand AODV routing protocols with DREAM and propose an location based routing protocol (EAODV_DREAM) to improve the route discovery. This proposed routing protocol can reduce the energy consumption of path failures and route discoveries. 


\section{NETWORK MODEL}

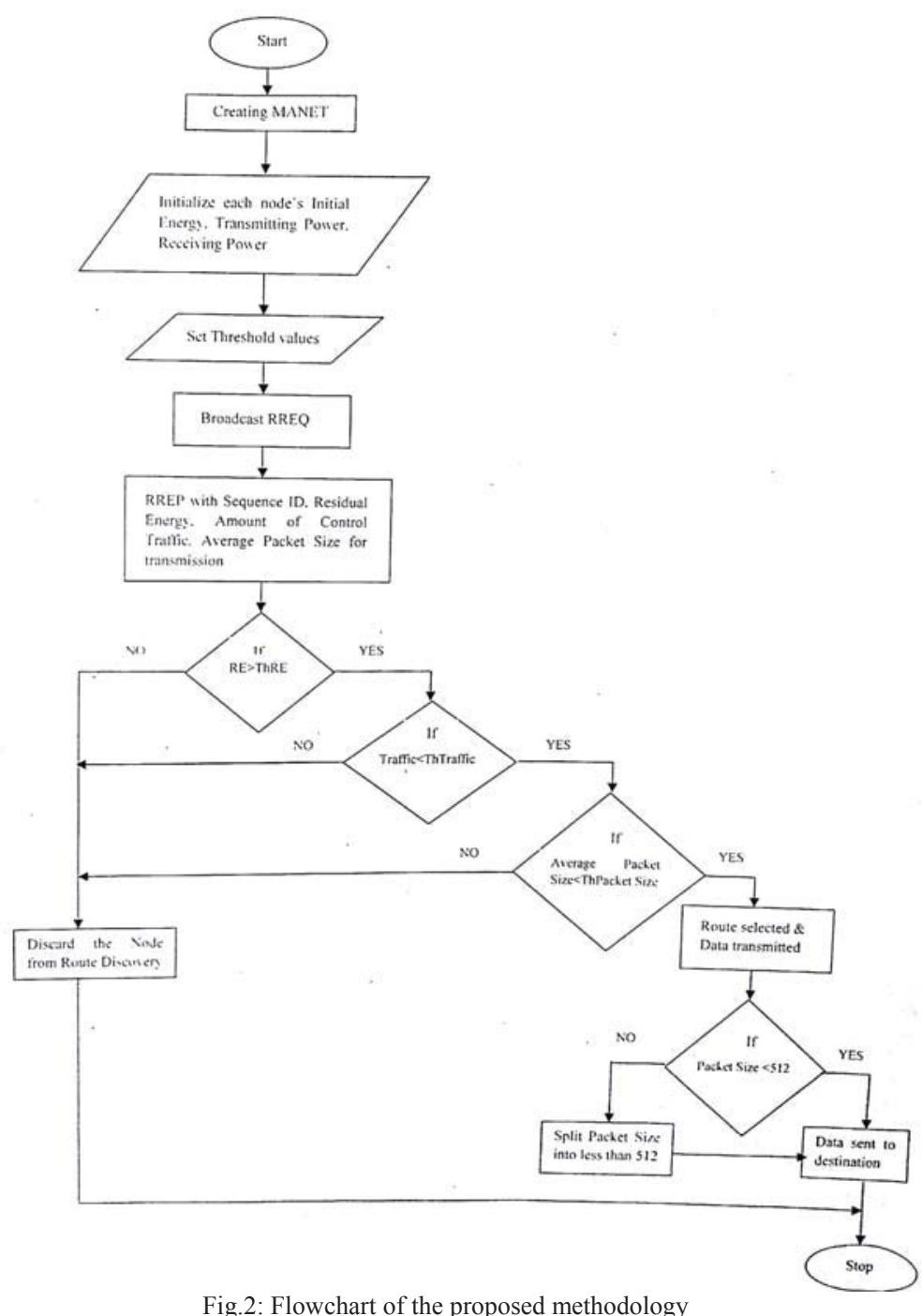

In our proposed methodology, the main goal is to conserve energy in each of the node. After creating the MANET all the nodes are initialized with some fixed transmitting power, receiving power sensing power and idle power. Depending on these initialized values, the consumed energy in each of the node is calculated. After that the total residual energy is calculated. Moreover, some threshold values are defined for Residual Energy, No. of control traffic, Average Packet Size that has already transmitted and Size of the Packet to be transmitted, which are used for choosing a node for communication, i.e., if a node's residual energy level is greater than the threshold residual energy value then it will check whether the no. of control traffic is less than the threshold value of no. of control traffic or not, if yes, then that node is involved in transmission or reception of data packets. Finally, the protocol will check whether the packet size to be transmitted is smaller than the threshold packet size or not; if yes, then the data packet is forwarded to the final destination; if no, then the data packet is split into the same size of threshold packet size as defined and forwarded the split up data packets to the final destination.

\section{IMPLEMENTATION \& ANALYSIS}


In our proposed methodology, energy conservation is the main motive. For this NS2 is used for implementation. First step is to create a MANET with some number of nodes. For creating the MANET, the following are the defined options for NS2 code:

a. NS2 Parameter setting:

In our proposed methodology, we are setting the some parameters as constant, for creating MANET. We are considering the MANET with 27 numbers of nodes which is based on only the AODV Routing Protocol. The area for creating the MANET is of 500*500. The channel for creating Ad-hoc network is set as Wireless channel, radiopropagation model is set as Two Ray Ground, Network interface type is of wireless. For creating MANET, 802.11 is the MAC type. The antenna used in our methodology is of Omni Antenna.

Table I. Simulation Parameter

\begin{tabular}{|c|c|}
\hline Area & $500 \times 500$ \\
\hline No. of Nodes & 27 \\
\hline Simulation time & $160 \mathrm{~ms}$ \\
\hline Traffic Type & CBR \\
\hline Packet Size & AODV \\
\hline Routing Protocol & 50 \\
\hline Antenna model & Antenna/Omni Antenna \\
\hline Maximum packets in Ifq & 10 watt \\
\hline Initial Energy & 5 watt \\
\hline Transmitting Power & 3 watt \\
\hline Receiving Power & ON \\
\hline Sense Power & \\
\hline Movement Trace & \\
\hline
\end{tabular}

b. Create Energy Details trace File:

set en [open energyconsumption.tr w]

c. Formulas used to calculate the Energy level of nodes:

Transmitted Energy: It is the energy required to transmit data packets in each of the node [14].

ETx $=($ Transmitted Power $\times$ Packet Size $) / 2 \times 10^{6}$

where ETx is the transmitted Energy

Receiving Energy: It is the energy required to receive data packets in each of the node [14].

$\mathrm{ERx}=($ Receiving Power $\mathrm{x}$ Packet Size $) / 2 \times 10^{6}$

where ERx is the receiving energy

Consumed Energy: It is the total energy spent on transmitting and receiving packets in the MANET [14].

Consumed Energy=Energy Tx + Energy $\mathrm{Rx}$

Residual Energy: It is the total remaining energy after transmitting and receiving the packets in the MANET [14].

Residual Energy=Initial Energy-Consumed Energy

Finally Total Residual energy is calculated.

In our proposed methodology, transmission power and receiving power are taken as constant. Only the variable parameter is packet size. Therefore the calculation of Residual Energy of a node is based mainly on the packet size.

d. Simulation Result:

The Basic AODV is first implemented and run on given MANET scenario in NS-2 and energy level of each of the is calculated. The simulation result of the calculation of energy level of each of the node is given in Fig. 4: 


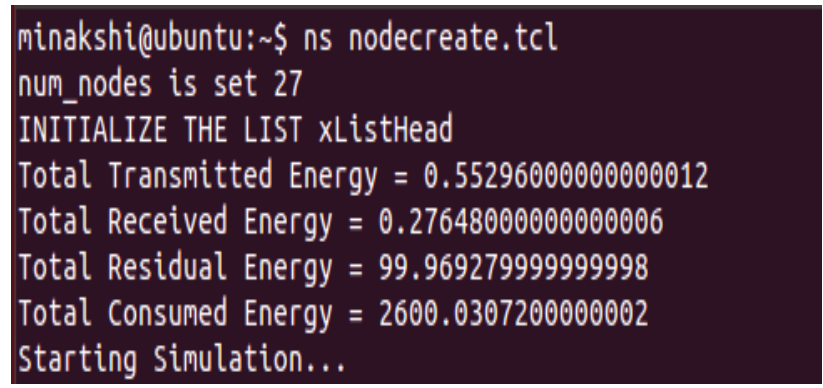

Fig. 4. Simulation result of Energy Calculation using NS2

After simulating the NS2 code, a nam (Network Animator) file generated. The output of nam file is given in Fig.4.

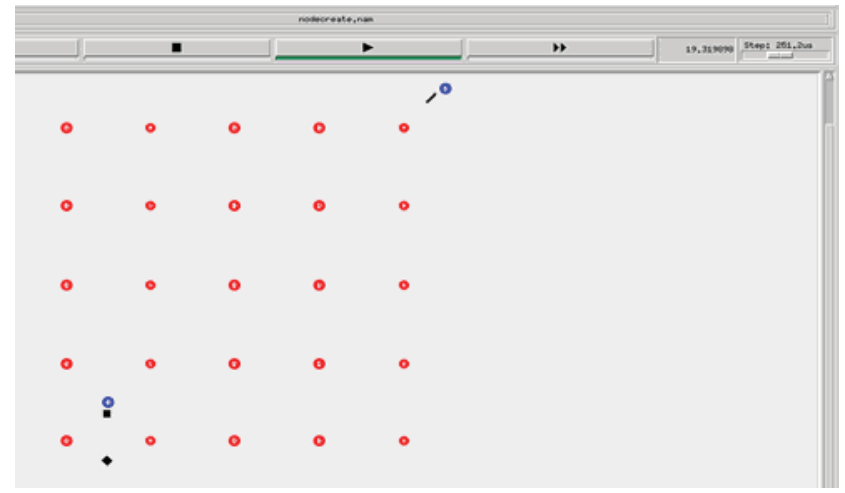

Fig. 4. Outputs of nam file (nodecreate.nam)

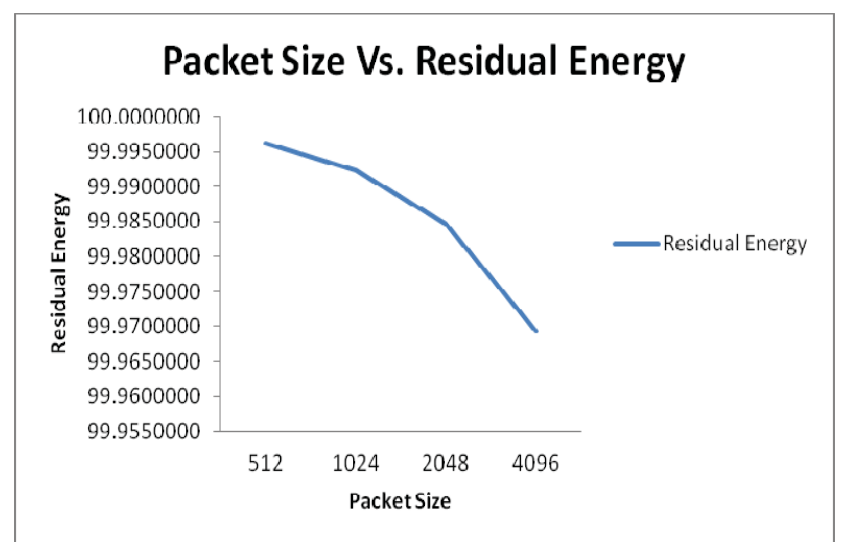

Fig. 5. Graphical representation of Residual Energy Vs. varying Packet Size

From the above graph it is found that the energy level in each of the node is gradually decreasing with the increase of packet size. In the simulation, the packet size is gradually increasing from 512 byte up to 4096 byte. That is with the value of increased packet size, the corruption of data bits may occur as a result of which the rate of retransmission increases. This will lead to consume more energy, so the residual energy is also decreased. This will affect the lifetime of a node in MANET [15].

\section{CONCLUSION AND FUTURE WORK}

In our proposed methodology the energy level of each of the node is calculated with respect to different packet size. Based on this calculation it is found that with the increase of packet size, the residual energy of each of the node is decreasing gradually as shown in Fig. 5. Based on this analysis, the proposed algorithm is designed. For implementing this algorithm, these files are modified: aodv.h, aodv.cc and energy-model.cc. It helps in calculating the residual energy of each of node in the MANET. However, the energy level can be minimized by calculating the average no. of control packet as well as minimizing the packet size less than 512 bits. 


\section{REFERENCES}

[1] Ravi, G., and K. R. Kashwan. "A new routing protocol for energy efficient mobile applications for ad hoc networks." Computers \& Electrical Engineering (2015).

[2] García Villalba, Luis Javier, et al. "Auto-configuration protocols in mobile ad hoc networks." Sensors 11.4 (2011): 3652-3666.

[3] Gopinath, S., and N. Nagarajan. "Energy based reliable multicast routing protocol for packet forwarding in MANET." Journal of applied research and technology 13.3 (2015): 374-381.

[4] Sridhar, S., R. Baskaran, and P. Chandrasekar. "Energy supported AODV (EN-AODV) for QoS routing in MANET." Procedia-Social and Behavioral Sciences 73 (2013): 294-301.

[5] Sumathi, K., and A. Priyadharshini. "Energy Optimization in Manets Using On-demand Routing Protocol." Procedia Computer Science 47 (2015): 460-470.

[6] Elavarasipriyanka, S., and V. Latha. "Design of Routing Protocol For MANET Through Energy Optimization Technique." Energy (2015).

[7] Feeney, Laura Marie, and Martin Nilsson. "Investigating the energy consumption of a wireless network interface in an ad hoc networking environment." INFOCOM 2001. Twentieth Annual Joint Conference of the IEEE Computer and Communications Societies. Proceedings. IEEE. Vol. 3. IEEE, 2001.

[8] Toh, Chai-Keong. "Maximum battery life routing to support ubiquitous mobile computing in wireless ad hoc networks." Communications Magazine, IEEE39.6 (2001): 138147.

[9] Tanque, Mohammed, and Rumana Islam. "Minimum Energy Dynamic Source Routing Protocol for Mobile Ad Hoc Networks." (2007).

[10] Baisakh, Baisakh. "A Review of Energy Efficient Dynamic Source Routing Protocol for Mobile Ad Hoc Networks." International Journal of Computer Applications 68.20 (2013): 6-15

[11] Mohamed Sharifdeen. M, Dhavamaniprakash. D. "An Energy Efficient DSR Routing Protocol for Energy Consumption in MANET", International Journal of Innovative Research in Science, Engineering and Technology, ISO 3297: 2007

[12] Agrawal, Aishwary, Damodar Tiwari, and Shailendra Singh. "LEACH-DSR Base Routing For Minimization Energy Consumption in MANET." International Journal of Scientific and Research Publications: 370.

[13] Rai, Avinash, and Tripti Arjariya. "Energy Efficient Ad hoc on Demand DREAM Routing in Wireless Ad Hoc Network." International Journal of Multidisciplinary Approach \& Studies 2.1 (2015).

[14] Kumar, Ashish, M. Q. Rafiq, and Kamal Bansal. "Performance Evaluation of Energi consumption in MANET." International Journal of Computer Applications (0975-8887) 42.2 (2012).

[15] Sharma, Namita. "Impact of Varying Packet Size on multihop routing protocol in Wireless Sensor Networks." International Journal of Advanced Studies in Computers, Science and Engineering 3.9 (2014): 10. 